\title{
Psychometric Evaluation of the Diabetes Distress Scale in Patients with Type 2 Diabetes in Turkey
}

\author{
Nurten Terkes $^{1^{*}}$ (D), Hicran Bektas ${ }^{2}$ (D)
}

\begin{abstract}
The objective of the research was to evaluate the validity and reliability of the Diabetes Distress Scale in patients with type 2 diabetes in Turkey.

Materials and Methods. Our study was conducted between September 2016 and January 2017 and included 170 patients with type 2 diabetes. The Personal Information Form and Diabetes Distress Scale were used as a data collection tool. Statistical analysis was performed using SPSS 23.0 and SAS package program.

Results. According to the results of the research, Cronbach's alpha reliability for the total scale was 0.91. The model fit indices for the revised confirmatory factor analysis model failed to meet the criteria for acceptability: the GFI was 0.8185, the CFI was 0.9316, the Bentler - Bonett (1980) NFI was 0.9005, and the RMSEA was 0.1067. In our study, exploratory factor analysis provided support for the three-factor model: [I] emotional and regimen-related distress, [II] health professional-related distress, [III] diabetes-related interpersonal distress.

Conclusions. When the translation and cultural adaptation process have been considered, the Diabetes Distress Scale is a valid and reliable tool for the Turkish community. It is recommended to be used in the studies and clinical trials.

Keywords

Type 2 Diabetes; Reliability; Validity; Diabetes Distress Scale

${ }^{1}$ Department of Internal Medicine Nursing, Bucak Health School, Mehmet Akif Ersoy University, Burdur, Turkey

${ }^{2}$ Department of Internal Medicine Nursing, Faculty of Nursing, Akdeniz University, Antalya, Turkey

*Corresponding author: nurtenterkes@gmail.com
\end{abstract}

Copyright $\odot$ Nurten Terkes, Hicran Bektas, 2

\section{Introduction}

Diabetes mellitus (DM) is a disease that develops as a result of insulin resistance, decreased production and use of insulin, and is associated with poor lifestyle choices and obesity [1]. According to the International Diabetes Federation (IDF), in 2019, the prevalence of diabetes among the global adult population reached $9.3 \%$; there were approximately 463 million individuals with diabetes; approximately 4.2 million individuals died from diabetes and its complications [2]. According to the results of the Turkish DM Epidemiology Study (TURDEP-II), the prevalence of DM was found to be $13.7 \%$ [3].

A lot of people still fail to meet the recommended treatment goals, despite improved technologies, new oral medications, and better insulin. Therefore, patients may feel exhausted, overwhelmed, or fed up. Such problems have been reported to negatively affect blood glucose control and quality of life [4-6]. Organizations such as the American Diabetes Association and the International Diabetes Federation recommend developing tools to detect such psy- chological problems [7, 8].

Emotional burdens and concerns related to the management of diabetes, risk of complications, unmet needs from healthcare providers, friends, and family are defined as diabetes distress [5, 9]. Diabetes distress is associated with higher glycosylated hemoglobin (HbA1c) test [10] and lower diabetes-related quality of life [11]. In addition, untreated psychological disorders such as stress and distress can lead to cardiovascular problems, depression, and cognitive decline $[12,13]$. In addition, a study has found that participants with uncontrolled HbA1c had significantly higher mean Diabetes Distress Scale (DDS) scores with a significant positive correlation between the last measured HbA1c level and the number of ketoacidosis attacks and DDS scores [14]. These findings highlighted the importance of routine screening for diabetes distress and clinically significant depressive symptoms [11].

Therefore, valid and reliable instruments for assessing diabetes distress are needed to discriminate between the levels of diabetes-related emotional problems and those who are clinically depressed. Many scales with differ- 
ent contents have been developed for the holistic evaluation of individuals with diabetes [15]. Having scales that identify individual needs provides support for designing effective educational interventions, as well as promoting collaborative interventions in the fight against disease [16]. The DDS has been used in different cultures and clinical practices [8, 17-19]. In the literature, validity and reliability studies of the scale have been conducted in many countries such as China [17], Norway [18], and Denmark [20]. No scale in Turkey can assess the level of distress related to diabetes.

Therefore, the objective of our research was to make the validity and reliability of the DDS-17 scale for Turkish society.

\section{Materials and Methods}

Initially, the validity and reliability studies were carried out within the scope of the Turkish adaptation of the original scale, by examining the psycholinguistic structure (language adaptation) and the psychometric structure (validityreliability), comparing cross-cultural characteristics. Within the scope of validity, language validity, content validity, and construct validity were made. In the language validity phase, the translation (English-Turkish) and backtranslation (Turkish-English) methods were used. Within the scope of construct validity, confirmatory and exploratory factor analyzes of the data were made. Internal consistency and item analysis were evaluated within the scope of reliability. Internal consistency analysis was determined by the Cronbach's alpha coefficient; item analysis was determined by Pearson product-moment correlation.

\section{Design and Sample}

We invited patients with type 2 diabetes to participate in this cross-sectional survey study by visiting an endocrinology outpatient clinic at University Hospital in Turkey between September 15, 2016, and January 15, 2017. Inclusion criteria were as follows: 1) 18 years of age or older; 2) diagnosis of diabetes with disease onset $\geq 1$ year; 3 ) absence of any psychiatric disorder; 4) accepting to participate in the study.

Participants were verbally informed of the purpose and protocols of the study, the time required for their participation, and that they could refuse if they wished. The sample size was calculated based on the participant ratio of 1:5 or 1:10 [20] items in the DDS-17. The DDS-17 has 17 elements. The minimum number of participants required to better realize the dimensionality of the instrument is $17 * 10=170$.

\section{Data Instruments \\ Demographics}

A basic demographic questionnaire was used to collect descriptive data of the participants (such as age, gender, occupation status, education, employment status, illness duration, treatment methods, other chronic diseases, body mass index.)

\section{Diabetes Distress Scale-17}

The original 17-item DDS was tested by Polonsky et al. on patients with type 1 and 2 in both primary care and specialist settings [21]. The DDS-17 was developed in 2005 to overcome limitations of previous measures of diabetes distress. The total items were divided into four subscales, covering the life of diabetic patients: emotional burden (5 items); physician-related distress (4 items); regimenrelated distress (5 items); diabetes-related interpersonal distress ( 3 items). The response to each item was based on a 6-point Likert scale, rated from 1 (not a problem) to 6 (a very serious problem) concerning diabetes over the past 1 month; thus, higher values indicated greater distress [21]. A mean item score of $\geq 3$ (high distress) was considered as a level of distress worthy of clinical attention, whereas an overall mean score of $<2.0$ indicated "little to no distress", a score between 2.0 and 2.9 indicated "moderate distress" [21-24]. The original version of the DDS-17 is reliable (Cronbach's alpha $=0.93$ ) [21].

\section{Translation Procedure}

First, the Turkish translation of the original scale was made (Fig. 1). It was performed independently by two

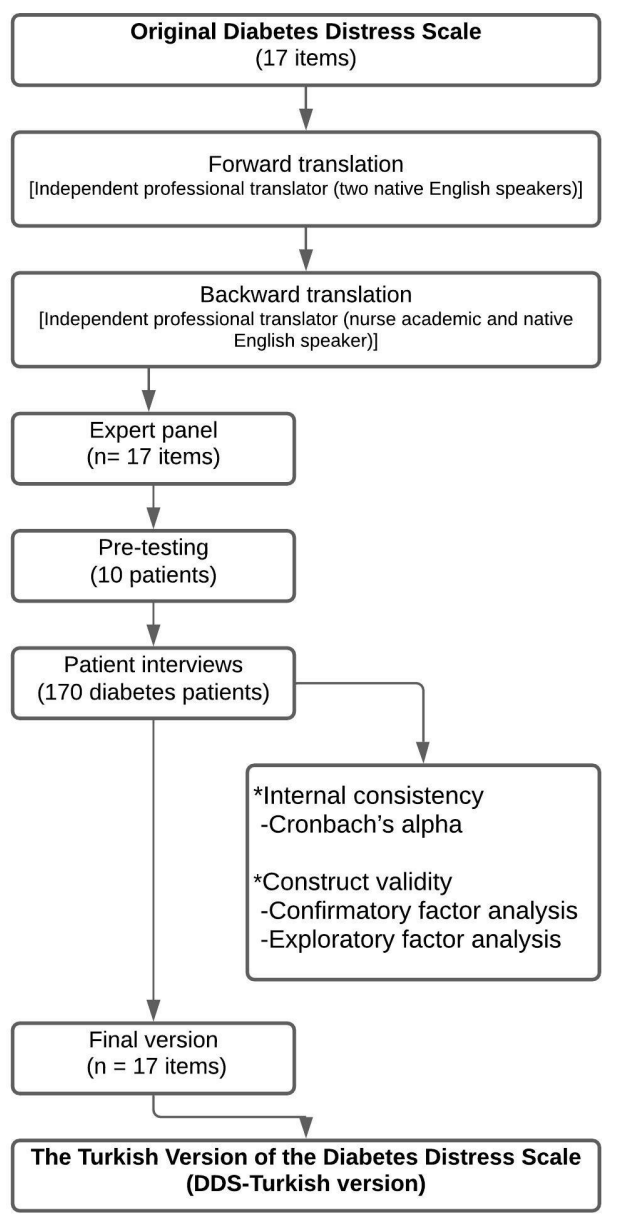

Figure 1. Flowchart depicting the process used for translation, validation and item evaluation of the instrument. 
translators who are fluent in both English and Turkish; one of them was a medical officer in practice as a general practitioner and the other one was a qualified linguistic translator. Later, the Turkish version was translated back into the original language. This process was carried out by another two translators, a general practitioner, and a qualified linguistic translator. In the last step of the translation procedure, the Turkish version was reviewed by an expert panel: professional translator, medical specialist in diabetes, nutritionist, two nurses with diabetes experience in the adaptation and validation of tools. In addition, two researchers took part. A final version ready to be tested was created.

After modification to the terminology, the Turkish version was applied to 10 Turkish patients who met the study criteria. Independent classifications of the DDS-17-Turkish (DDS-17-T) items yielded a good inter-rater agreement (Kendall's W $=0.65$ ), and the consensus discussion yielded a full agreement. Participants were explained the purpose of the study. In addition, they were informed if they had difficulties in understanding the items of the scale. Participants did not report any problems. The findings of these individuals were not included in the validity and reliability studies (Fig. 1).

\section{Statistical Analysis}

Statistical analysis was carried out by using the IBM SPSS statistical program (v. 23.0; IBM Corporation, Armonk, NY, USA) with a significance level of $p<0.05$. Three methods were used to test the psychometric properties of the DDS-17-T, namely factor analysis, item analysis, and internal consistency. Confirmatory factor analysis (CFA) was first conducted on this study sample to confirm the exploratory model developed by Polonsky et al. (2005) [21]. If the model fit is unsatisfactory, then, efforts to respecify the model, using modification indices, are completed and the revised model is re-evaluated with CFA. If the revised model continues to demonstrate poor fit, then, exploratory factor analysis (EFA) is used to examine the factor structure to determine if there is an empirical basis to revise the subscales of the test. In these model fit criteria, a model is suitable if the goodness of fit index (GFI) value is greater than 0.9 , the adjusted goodness of fit index (AGFI) value is greater than 0.8 , the root mean square error of approximation (RMSEA) value is less than 0.08, and the comparative fit index (CFI) and non-normed fit index (NNFI) values are greater than 0.95 [25, 26]. Before EFA, the Kaiser-MeyerOlkin (KMO) value was analyzed to determine whether the sample was adequate or not, and the Bartlett test was used to determine whether the correlation matrix was a unit matrix, indicating a rationale for factor analysis. In the literature, factor loading of $\geq 0.40$ is recommended for each item [27]. We used Cronbach's alpha to determine internal consistency for the DDS-17-T total scores and DDS-17-T subscales.

\section{Results}

\section{Sample Characteristics}

In this study, 170 patients with type 2 diabetes were interviewed. The average HbA1c value was 7.0 ( $\mathrm{SD}=1.38$ ). The mean age was 58.9 years; the duration of diabetes was 2.1 years; $62.4 \%$ of the patients were females. Most of the participants $(80 \%)$ were receiving oral medication (either monotherapy or combinations of two or three oral antidiabetic drugs), and a total of $41.2 \%$ had obesity. Sociodemographic characteristics and clinical conditions of the participants are shown in Table 1.

Table 1. Demographic and medical characteristics of the patients with type 2 diabetes $(n=170)$.

\begin{tabular}{|c|c|c|}
\hline & Mean \pm SD & Range \\
\hline HbA1c (\%) & $7.0 \pm 1.38$ & $5.3-12.9$ \\
\hline Age (years) & $58.9 \pm 1.02$ & $20-65$ \\
\hline \multirow[t]{2}{*}{ Illness duration (years) } & $2.1 \pm 0.78$ & $1-25$ \\
\hline & $\mathbf{n}$ & $\%$ \\
\hline \multicolumn{3}{|l|}{ Gender } \\
\hline Female & 106 & 62.4 \\
\hline Male & 64 & 37.6 \\
\hline \multicolumn{3}{|l|}{ Marital status } \\
\hline Married & 158 & 92.9 \\
\hline Single & 12 & 12.1 \\
\hline \multicolumn{3}{|l|}{ Occupational status } \\
\hline Housewife & 73 & 42.9 \\
\hline Retired & 69 & 40.6 \\
\hline Public servant & 19 & 11.2 \\
\hline Self-employed & 9 & 5.3 \\
\hline \multicolumn{3}{|l|}{ Education } \\
\hline Illiterate & 21 & 7.2 \\
\hline Primary & 81 & 47.6 \\
\hline Secondary & 14 & 8.2 \\
\hline High & 30 & 17.6 \\
\hline University & 33 & 19.4 \\
\hline \multicolumn{3}{|l|}{ Employment status } \\
\hline Unemployed & 143 & 84.1 \\
\hline Employed & 27 & 15.9 \\
\hline \multicolumn{3}{|l|}{ Duration of the illness } \\
\hline$<5$ year & 47 & 27.6 \\
\hline $6-10$ year & 66 & 38.8 \\
\hline$>10$ year & 57 & 33.6 \\
\hline \multicolumn{3}{|l|}{ Treatment methods } \\
\hline Medical nutrition therapy & 340 & 100 \\
\hline Oral antidiabetic agents & 136 & 80 \\
\hline Exercises & 100 & 58.8 \\
\hline Insulin injections & 50 & 29.4 \\
\hline Antidiabetic agents+insulin & 16 & 9.4 \\
\hline \multicolumn{3}{|l|}{ Other chronic diseases } \\
\hline Hypertension & 79 & 46.5 \\
\hline Thyroid diseases & 35 & 20.6 \\
\hline Cardiovascular disease & 34 & 20 \\
\hline \multicolumn{3}{|l|}{ Body mass index } \\
\hline 18.5 - 24.9 (normal weight) & 25 & 14.7 \\
\hline $25-29.9$ (overweight) & 63 & 37.1 \\
\hline $30-39.9$ (obesity) & 70 & 41.2 \\
\hline $40>$ (morbid obesity) & 12 & 7.0 \\
\hline
\end{tabular}




\section{Factorial Validity}

The validation study of this scale was carried out using CFA and EFA. First, CFA was performed in the SAS program for construct validity of the DDS-17-T. All model fit indices for the revised CFA model narrowly failed to meet the criteria for acceptability: the GFI was 0.8185 $\left(\chi^{2}(109)=318.7381, \mathrm{p}<0.0001\right)$, the CFI was 0.9316, the Bentler - Bonett (1980) NFI was 0.9005, and the RMSEA was 0.1067 (90\% lower confidence limit) (Table 2). The Bentler's CFI was just below acceptable one, indicating moderate fit. These results indicated that the fit between the model and the data required improvement, as well as the need for EFA modification of the model.

Table 2. Goodness of fit indices for the Diabetes Distress Scale - Turkish version

\begin{tabular}{lc}
\hline Index & Values \\
\hline GFI & 0.8185 \\
AGFI & 0.7453 \\
Chi-Square & 318.7381 \\
Chi-Square DF & 109 \\
Pr $>$ Chi-Square & 0.0001 \\
RMSEA Estimate & 0.1067 \\
Bentler's CFI & 0.9316 \\
Bentler - Bonett (1980) NFI & 0.9005 \\
\hline
\end{tabular}

In the second stage, EFA was conducted. The results of EFA showed a KMO value of 0.88 , indicating sample adequacy for factor analysis. The Bartlett's test of sphericity was significant $\left(\chi^{2}=3078, p<0.001\right)$. This result showed that the data were suitable for further analysis. Factor 1 (emotional and regimen-related distress) $(40.19 \%)$ included ten items $(2,3,4,6,7,8,10,12,14,16)$; Factor 2 (health professional-related distress) (20.21\%) included four items $(1,5,11,15)$; Factor 3 (interpersonal distress) $(16.84 \%)$ included three items $(9,13,17)$. The emotional distress and regime distress subscales were combined as one factor. The other subscales were completely similar to the original subscales. All components explained $77.25 \%$ of the total variance (Table 3, Fig. 2).

The component correlation matrix presents the correlation between the extracted factors/components and is, therefore, important for choosing between vertical and oblique rotation. In our study, the factor correlation matrix of the subscales is given in Table 4 .

\section{Internal Consistency Reliability}

Internal consistency is evaluated using the Cronbach's alpha coefficient to determine whether all items in a multiitem scale measure the same concept. The Cronbach's alpha value $>0.70$ has sufficient internal consistency [28]. In our study, the Cronbach's alpha value was calculated for the whole instrument and each subscale. Following the three patterns from the EFA in this study, Cronbach's alpha was 0.94 for the combined emotional and regimenrelated distress subscale, 0.92 for the health professionalrelated distress subscale, and 0.96 for the diabetes-related interpersonal distress subscale. These alpha values were

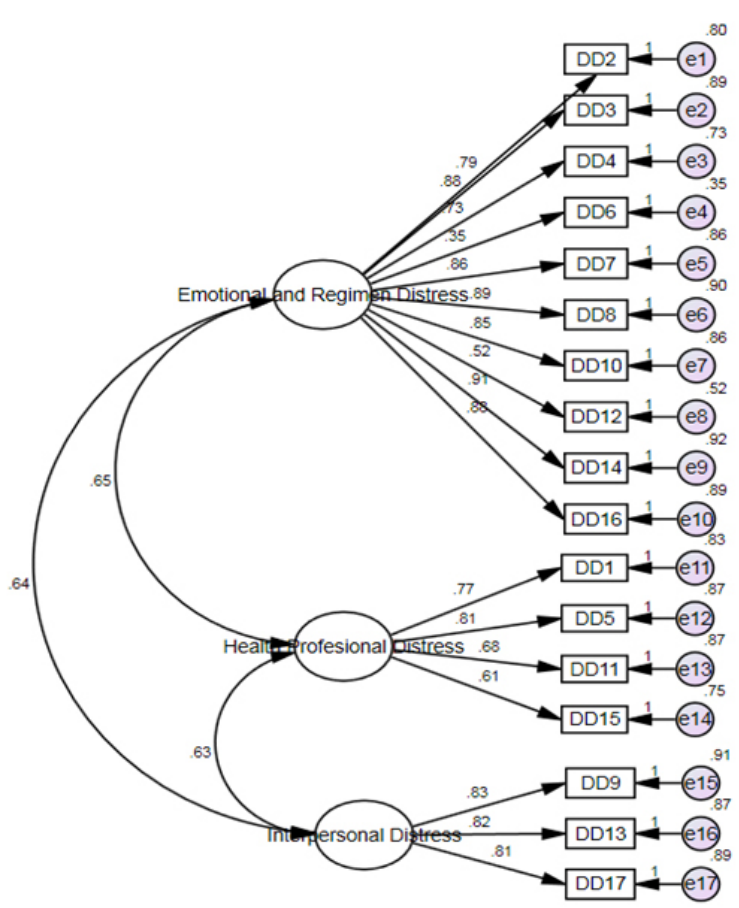

Figure 2. Confirmatory Factor Analysis.

sufficient, especially considering the number of items per scale (Table 5).

\section{Discussion}

The validity and reliable scales are needed to reveal diabetesrelated emotional distress. The DDS was developed in English to identify diabetes-related emotional distress and areas of concern [21, 29]. It is seen in the literature that adaptations to many languages have been made $[17,18,20,30]$. The main objective of this paper was to report the reliability and validity of the DDS-17-T in a sample of adult Turkish patients with type 2 diabetes.

In the Turkish translation of the original scale, the expression "health professionals" was used instead of doctors only in items $1,5,11$, and 15 . Otherwise, no changes were made. Nurses play an important role in promoting patients' self-care. Not only language translation is needed to apply an existing scale for an intercultural study, cultural and environmental contexts of equal importance need to be considered as well [31]. This was similar to the Thai DDS-17 validation study in Thailand, where the "physician distress" domain was also modified and renamed "physician- and nurse-related distress" [32].

In our study, EFA supported the three-factor model rather than the four-factor model in the original DDS-17. The factor structure of the DDS-17-T differs from that of the original DDS-17 in that emotional burden and regimerelated distress are included in the same factor. This factor structure is different from the original scale that has four factors but is similar to the DDS-17 validated in China (CDDS) [17] and Thailand (Thai DDS-17) [32]. This difference can be attributed to the cultural differences of Turkish patients who think that lifelong treatment and self- 
Table 3. Factor loading, pattern matrix and structure matrix of the Diabetes Distress Scale - Turkish version $(\mathrm{n}=170)$.

\begin{tabular}{|c|c|c|c|c|c|c|}
\hline & \multicolumn{6}{|l|}{ ITEMS } \\
\hline & Factor 1 (Emotional and regimen-related distress) & F1 & $\mathbf{F} 2$ & F3 & $\begin{array}{c}\text { Pattern } \\
\text { Ma- } \\
\text { trix }\end{array}$ & $\begin{array}{l}\text { Structure } \\
\text { Matrix }\end{array}$ \\
\hline$(2)$ & $\begin{array}{l}\text { Feeling that diabetes is taking up too much mental and physi- } \\
\text { cal energy every day. }\end{array}$ & 0.798 & -0.278 & -0.097 & 1.085 & 0.806 \\
\hline (3) & $\begin{array}{l}\text { Feeling not enough confident in my day-to-day ability to man- } \\
\text { age diabetes. }\end{array}$ & 0.883 & -0.299 & -0.112 & 1.195 & 0.892 \\
\hline (4) & $\begin{array}{l}\text { Feeling angry, scared and/or depressed when I think about } \\
\text { living with diabetes. }\end{array}$ & 0.733 & -0.209 & -0.091 & 0.953 & 0.739 \\
\hline$(6)$ & $\begin{array}{l}\text { Feeling that I am not testing frequently enough my blood } \\
\text { sugars. }\end{array}$ & 0.351 & -0.018 & -0.001 & 0.357 & 0.350 \\
\hline$(7)$ & $\begin{array}{l}\text { No matter what I do, I feel that I will end up with serious } \\
\text { long-term complications. }\end{array}$ & 0.865 & -0.116 & -0.004 & 0.952 & 0.869 \\
\hline$(8)$ & Feeling that I am often failing with my diabetes routine. & 0.899 & -0.203 & -0.037 & 1.086 & 0.905 \\
\hline (10) & Feeling that diabetes controls my life. & 0.854 & -0.246 & 0.000 & 1.069 & 0.862 \\
\hline (12) & Feeling that I am not sticking closely to a good meal plan. & 0.527 & 0.099 & -0.196 & 0.494 & 0.520 \\
\hline (14) & Feeling overwhelmed by the demands of living with diabetes. & 0.914 & -0.250 & -0.071 & 1.161 & 0.921 \\
\hline \multirow[t]{2}{*}{$(16)$} & $\begin{array}{l}\text { Feeling not motivated to keep up self-management about my } \\
\text { diabetes. }\end{array}$ & 0.889 & -0.194 & -0.080 & 1.084 & 0.894 \\
\hline & Factor 2 (Health profesional-related distress) & & & & & \\
\hline$(1)$ & $\begin{array}{l}\text { Feeling that my doctor does not know enough about diabetes } \\
\text { and diabetes care. }\end{array}$ & 0.340 & 0.774 & -0.337 & 1.205 & 0.831 \\
\hline$(5)$ & $\begin{array}{l}\text { Feeling that my doctor does not give me clear directions on } \\
\text { how to manage my diabetes. }\end{array}$ & 0.375 & 0.811 & -0.293 & 1.218 & 0.876 \\
\hline (11) & Feeling that my doctor does not take seriously my concerns. & 0.315 & 0.688 & -0.198 & 1.007 & 0.871 \\
\hline \multirow[t]{2}{*}{$(15)$} & $\begin{array}{l}\text { Feeling that I do not have a doctor whom I can visit regularly } \\
\text { concerning my diabetes. }\end{array}$ & 0.319 & 0.619 & -0.225 & 0.939 & 0.759 \\
\hline & Factor 3 (Interpersonal distress) & & & & & \\
\hline$(9)$ & $\begin{array}{l}\text { Feeling that friends or family do not support enough my self- } \\
\text { care efforts. }\end{array}$ & 0.378 & 0.311 & 0.831 & 1.220 & 0.913 \\
\hline (13) & $\begin{array}{l}\text { Feeling that my friends or my family do not appreciate how } \\
\text { difficult living with diabetes can be. }\end{array}$ & 0.347 & 0.263 & 0.826 & 1.202 & 0.872 \\
\hline (17) & $\begin{array}{l}\text { Feeling that my friends or my family do not give me emotional } \\
\text { support that I would like to have. }\end{array}$ & 0.404 & 0.241 & 0.819 & 1.187 & 0.896 \\
\hline
\end{tabular}

Notes: Extraction Method: Principal Axis Factoring. Rotation method: Oblimin with Kaiser Normalization.

Table 4. Factor Correlation Matrix.

\begin{tabular}{l|ccc}
\hline Factor & $\begin{array}{c}\text { Emotional and } \\
\text { regimen-related } \\
\text { distress }\end{array}$ & $\begin{array}{c}\text { Health } \\
\text { professional-related } \\
\text { distress }\end{array}$ & Interpersonal distress \\
\hline Emotional and regimen-related distress & 1.000 & 1.000 & 1.000 \\
Health professional-related distress & 0.652 & 0.634 & 1 \\
Interpersonal distress & 0.643 & 0 & \\
\hline
\end{tabular}

Notes: Extraction Method: Principal Axis Factoring. Rotation method: Oblimin with Kaiser Normalization.

Table 5. Mean, standard deviation (SD), internal consistency (Cronbach's $\alpha$ coefficient) of the Diabetes Distress Scale Turkish version for patients with type 2 diabetes $(n=170)$.

\begin{tabular}{lccccc}
\hline Scale & Total items & Range of scores & Mean & SD & $\alpha$ \\
\hline Emotional and regimen-related distress & 10 & $10-59$ & 21.52 & 12.24 & 0.94 \\
Health professional-related distress & 4 & $4-24$ & 10.30 & 6.58 & 0.92 \\
Interpersonal distress & 3 & $3-17$ & 4.47 & 3.57 & 0.96 \\
Total & 17 & $17-88$ & 36.30 & 16.73 & 0.91 \\
\hline
\end{tabular}


management regimens are necessary for healthy life with diabetes, and this may be closely related to the emotional burden. In the study of Polonsky et al. (2005), the emotional burden and regime-related distress subscales of the original DDS-17 were highly correlated $(r=0.88)$, suggesting that the two subscales were not completely independent [21]. Similar to our study, the Thai DDS-17 validation study conducted in Thailand was a 3-factor model: emotional distress and regime distress were combined as one factor [32]. In addition, in the study of the Chinese population, the three factors for the DDS-17 validation study were an emotional burden, regime- and social support-related distress, and doctor-related distress [17]. It is thought that this difference might be due to the cultural difference in the experience of psychological problems.

Our study among Turkish patients with type 2 diabetes showed high internal consistency of the DDS-17-T (0.91), which is comparable to that of the original DDS17 (0.93) [19]. This result shows relatively similar internal consistency with results from cross-cultural studies of the DDS-17 scale [17, 18, 32, 33]. The findings from our study suggest that the DDS-17-T is suitable for using in daily clinical practice to identify and assess diabetes distress, especially in patients whose diabetes is poorly controlled.

\section{Limitations}

There are some limitations to our study. Firstly, the findings cannot be generalized as the sample was not randomly selected and the data were collected from only one institution. Secondly, the DSD-17-T includes only a sample of patients with type 2 diabetes; it has not been confirmed in patients with type 1 diabetes. Finally, it is worth evaluating whether the scale is sensitive to detecting emotional changes over time.

\section{Conclusions}

As diabetes requires strenuous routine care and self-management, it is important to identify and support psychological needs of individuals. The DDS-17-T gives satisfactory results in terms of the translation process and cultural adaptation and has acceptable psychometric properties. In conclusion, the DDS-17-T is a valid and reliable tool to identify diabetes-related distress in patients with type 2 diabetes and to identify the areas of problems. Future studies on larger samples are needed to implement interventions to minimize the burden of diabetes distress among patients with type 2 diabetes.

\section{Ethical Statement}

This study had been approved by the Ethics Committee for Clinical Research of the Medical Faculty at a university (Number: 122.AKD Date: 17.02.2016). The written permission was obtained from the Department of Endocrinology and Metabolic Disease in the university hospital. The permission to use the DDS-17 was obtained from William H. Polonsky via e-mail. Both written and verbal consent were obtained from all participants in the study.
The objective of the study was explained to the participants. It was informed that they could leave the study whenever they wanted. All procedures contributing to this work comply with the ethical standards of the relevant national and institutional guidelines and the Declaration of Helsinki.

\section{Informed Consent}

Informed consent was obtained from all the patients before they study.

\section{Conflict of Interest}

No conflict of interest has been declared by the authors.

\section{Financial Disclosure}

There is no financial support.

\section{References}

[1] Chaney D, Clarke A. Nursing care of conditions related to the endocrine system. In: Brady A-M, McCabe C, McCann M, editors. Fundamentals of medicalsurgical nursing a systems approach. Wiley Blackwell; 2014. pp. 298-325.

[2] International of Diabetes Federation. IDF Diabetes Atlas, 9th edn. Brussels, Belgium: 2019. Available from: https://www.diabetesatlas.org

[3] Satman I, Omer B, Tutuncu Y, Kalaca S, Gedik S, Dinccag N, et al. Twelve-year trends in the prevalence and risk factors of diabetes and prediabetes in Turkish adults. European Journal of Epidemiology. 2013;28(2):169-180. Available from: https://doi.org/10.1007/s10654-013-9771-5

[4] Wang R-H, Wu L-C, Hsu H-Y. A path model of healthrelated quality of life in type 2 diabetic patients: a cross-sectional study in Taiwan. Journal of Advanced Nursing. 2011;67(12):2658-2667. Available from: https://doi.org/10.1111/j.1365-2648.2011.05701.x

[5] Fisher L, Gonzalez JS, Polonsky WH. The confusing tale of depression and distress in patients with diabetes: a call for greater clarity and precision. Diabetic Medicine. 2014;31(7):764-772. Available from: https://doi.org/10.1111/dme.12428

[6] Nicolucci A, Kovacs Burns K, Holt RIG, Comaschi M, Hermanns N, Ishii H, et al. Diabetes attitudes, wishes and needs second study (DAWN2 ${ }^{\mathrm{TM}}$ ): cross-national benchmarking of diabetes-related psychosocial outcomes for people with diabetes. Diabetic Medicine. 2013;30(7):767-777. Available from: https://doi.org/10.1111/dme.12245

[7] American Diabetes Association. Standards of medical care in diabetes-2017: summary of revisions. Diabetes Care. 2016;40(Supplement 1):S4-S5. Available from: https://doi.org/10.2337/dc17-S003 
[8] Fisher L, Glasgow RE, Strycker LA. The relationship between diabetes distress and clinical depression with glycemic control among patients with type 2 diabetes. Diabetes Care. 2010;33(5):1034-1036. Available from: https://doi.org/10.2337/dc09-2175

[9] Gonzalez JS, Fisher L, Polonsky WH. Depression in diabetes: have we been missing something important? Diabetes Care. 2010;34(1):236-239. Available from: https://doi.org/10.2337/dc10-1970

[10] Luzuriaga M, Leite R, Ahmed H, Saab PG, Garg R. Complexity of antidiabetic medication regimen is associated with increased diabetes-related distress in persons with type 2 diabetes mellitus. BMJ Open Diabetes Research \& Care. 2021;9(1):e002348. Available from: https://doi.org/10.1136/bmjdrc-2021-002348

[11] Beverly EA, Osowik F. Clinically significant depressive symptoms and high diabetes distress in adults with type 1 and type 2 diabetes in Appalachian Ohio. Journal of Osteopathic Medicine. 2021;121(10):813-824. Available from: https://doi.org/10.1515/jom-20210091

[12] Skinner TC, Carey ME, Cradock S, Dallosso HM, Daly H, Davies MJ, et al. Depressive symptoms in the first year from diagnosis of type 2 diabetes: results from the DESMOND trial. Diabetic Medicine. 2010;27(8):965-967. Available from: https://doi.org/10.1111/j.1464-5491.2010.03028.x

[13] Sullivan MD, Katon WJ, Lovato LC, Miller ME, Murray $\mathrm{AM}$, Horowitz KR, et al. Association of depression with accelerated cognitive decline among patients with type 2 diabetes in the ACCORD-MIND trial. JAMA Psychiatry. 2013;70(10):1041-1047. Available from: https://doi.org/10.1001/jamapsychiatry.2013.1965

[14] Alyahyawi NY, Alrifay RM, Albadi NA, Alqahtani MY, Alzahrani RM, Nazer BA, et al. The impact of diabetes distress on the glycemic control among adolescents and youth with type 1 diabetes in two tertiary centers, Jeddah, Saudi Arabia. Cureus. 2021;13(8):e17557. Available from: https://doi.org/10.7759/cureus. 17557

[15] El Achhab Y, Nejjari C, Chikri M, Lyoussi B. Disease-specific health-related quality of life instruments among adults diabetic: a systematic review. Diabetes Research and Clinical Practice. 2008;80(2):171-184. Available from: https://doi.org/10.1016/j.diabres.2007.12.020

[16] Torres H de C, Souza ER, Lima MHM, Bodstein RC. Educational intervention for self-care of individuals with diabetes mellitus. Acta Paulista de Enfermagem. 2011;24(4):514-519. Available from: https://doi.org/10.1590/S0103-21002011000400011

[17] Ting RZW, Nan H, Yu MWM, Kong APS, Ma RCW, Wong RYM, et al. Diabetes-related distress and physical and psychological health in Chinese type 2 diabetic patients. Diabetes Care. 2011;34(5):1094-1096. Available from: https://doi.org/10.2337/dc10-1612

[18] Graue M, Haugstvedt A, Wentzel-Larsen T, Iversen MM, Karlsen B, Rokne B. Diabetes-related emotional distress in adults: reliability and validity of the Norwegian versions of the Problem Areas in Diabetes Scale (PAID) and the Diabetes Distress Scale (DDS). International Journal of Nursing Studies. 2012;49(2):174-182. Available from: https://doi.org/10.1016/j.ijnurstu.2011.08.007

[19] Curcio R, Lima MHM, Alexandre NMC. Diabetes mellitus related tools adapted and validated to Brazilian culture. Revista Eletrônica de Enfermagem. 2011;13(2):331-337. Available from: https://doi.org/10.5216/ree.v13i2.9476

[20] Joensen LE, Tapager I, Willaing I. Diabetes distress in type 1 diabetes-a new measurement fit for purpose. Diabetic Medicine. 2013;30(9):1132-1139. Available from: https://doi.org/10.1111/dme.12241

[21] Polonsky WH, Fisher L, Earles J, Dudl RJ, Lees J, Mullan J, et al. Assessing psychosocial distress in diabetes: development of the diabetes distress scale. Diabetes Care. 2005;28(3):626-631. Available from: https://doi.org/10.2337/diacare.28.3.626

[22] Beavers AS, Lounsbury JW, Richards JK, Huck SW, Skolits GJ, Esquivel SL. Practical considerations for using exploratory factor analysis in educational research. Practical Assessment, Research and Evaluation. 2013;18(6):1-13.

[23] Kong L, Cai Y, Mei G, Gu R, Zhang X, Qin Y, et al. Psychological status and diabetesrelated distress of Chinese type 1 diabetes patients in Jiangsu province, China. Journal of Biomedical Research. 2013;27(5):380-385. Available from: https://doi.org/10.7555/JBR.27.20120112

[24] Fisher L, Hessler DM, Polonsky WH, Mullan J. When is diabetes distress clinically meaningful?: Establishing cut points for the diabetes distress scale. Diabetes Care. 2012;35(2):259-264. Available from: https://doi.org/10.2337/dc11-1572

[25] Nakahara R, Yoshiuchi K, Kumano H, Hara Y, Suematsu H, Kuboki T. Prospective study on influence of psychosocial factors on glycemic control in Japanese patients with type 2 diabetes. Psychosomatics. 2006;47(3):240-246. Available from: https://doi.org/10.1176/appi.psy.47.3.240

[26] Özdamar K. Explanatory factor analysis. In: Özdamar K, editor. Scale and Test Development in Education, Health and Behavioral Sciences Structural Equation Modeling, IBM, SPSS, IBM SPSS AMOS and MINITAB Applied. April Bookstore; 2016. pp. 133-160. 
[27] Revicki D, Ganguli A, Kimel M, Roy S, Chen N, Safikhani S, et al. Reliability and validity of the work instability scale for rheumatoid arthritis. Value in Health. 2015;18(8):1008-1015. Available from: https://doi.org/10.1016/j.jval.2015.09.2941

[28] Streiner DL, Norman GR. Health measurement scales: A practical guide to their development and use. 5th ed. New York, NY: Oxford University Press; 2015.

[29] Borsa JC, Damásio BF, Bandeira DR. Cross-cultural adaptation and validation of psychological instruments: some considerations. Paidéia (Ribeirão Preto). 2012;22(53):423-432. Available from: https://doi.org/10.1590/S0103-863X2012000300014

[30] Ortiz MS, Baeza-Rivera MJ, Myers HF. Psychometric properties of the Diabetes Distress Scale [DDS] in a sample of Chilean diabetic patients. Terapia psicológica. 2013;31(3):281-286. Available from: https://doi.org/10.4067/S0718-48082013000300002

[31] Curcio R, Alexandre NMC, Torres $\mathrm{H}$ de C, Lima MHM. Translation and adaptation of the "Diabetes Distress Scale - DDS" in Brazilian culture. Acta Paulista de Enfermagem. 2012;25(5):762-767.
Available from: https://doi.org/10.1590/S010321002012005000025

[32] Thanakwang K, Thinganjana W, Konggumnerd R. Psychometric properties of the Thai version of the Diabetes Distress Scale in diabetic seniors. Clinical Interventions in Aging. 2014;2014(9):1353-1361. Available from: https://doi.org/10.2147/CIA.S67200

[33] Tol A, Baghbanian A, Sharifirad G, Shojaeizadeh D, Eslami A, Alhani F, et al. Assessment of diabetic distress and disease related factors in patients with type 2 diabetes in Isfahan: A way to tailor an effective intervention planning in Isfahan-Iran. Journal of Diabetes \& Metabolic Disorders. 2012;11(1):20. Available from: https://doi.org/10.1186/2251-6581-11-20

Received: 2021-08-16

Revision Requested: 2021-10-05

Revision Received: 2021-10-14

Accepted: $2021-10-18$ 